\title{
Neuronal correlates of familiarity-driven decisions in artificial grammar learning
}

\author{
C. M. Thiel, ${ }^{1,5, C A}$ D. R. Shanks, ${ }^{2}$ R. N. A. Henson ${ }^{1,3}$ and R. J. Dolan ${ }^{1,4}$
}

\begin{abstract}
'Wellcome Department of Imaging Neuroscience, Institute of Neurology, 12 Queen Square, London, WCIN 3BG; ${ }^{2}$ Department of Psychology, University College London, Gower St, London WCIE 6BT; ${ }^{3}$ Institute of Cognitive Neuroscience, University College London, 17 Queen Square, London WCIN $3 A R$; ${ }^{4}$ Royal Free Hospital School of Medicine, London NW3 2PF, UK; Institute of Medicine, Cognitive Neurology Group, Research Centre Juelich, 52425 Juelich, Germany

CA,5 Corresponding Author and Address: c.thiel@fz-juelich.de
\end{abstract}

Received I3 November 2002; accepted 22 November 2002

DOI: 10.1097/0l.wnr.0000051003.67742.bc

\begin{abstract}
It has been proposed on the basis of behavioural data that grammaticality judgments in implicit artificial grammar learning paradigms are largely driven by priming based on fragment familiarity. A prediction that follows from this account is that neural deactivation, a common correlate of repetition priming, should be observed for grammatical compared to ungrammatical stimuli. We conducted an event-related fMRI study to investigate neuronal correlates of such fragment-based priming. In a study phase, participants performed a short-term memory task on a series of strings of pseudofont characters. Scanning was performed in a subsequent test phase in which participants classified new strings as either
\end{abstract}

grammatical or ungrammatical. Test strings differed systematically from training strings in terms of exemplar and fragment similarity. Behaviourally, participants classified strings as grammatical based on fragment familiarity. Differential activity was evident during string classification as reduced activity in left lateral occipital complex and bilateral lingual gyri for strings with high fragment familiarity compared to strings with low fragment familiarity. Thus, consistent with the hypothesis, neuronal facilitation in extrastriate occipital regions may constitute one basis of implicit grammaticality decisions based on fragment priming. NeuroReport $14: 131-136$ (c) 2003 Lippincott Williams \& Wilkins.

Key words: Artificial grammar; Categorization; Extrastriate cortex; Human; Implicit learning; Magnetic resonance imaging

\section{INTRODUCTION}

Exposure to a series of items enables us to classify new items as belonging to the same category. Category learning is often investigated in artificial grammar learning paradigms where volunteers are exposed to a series of letter strings, generated by an artificial grammar, and subsequently asked to classify new letter strings as either conforming to the grammar or not [1]. This type of learning has been the subject of great interest as it has been claimed to occur implicitly, or unconsciously [1]. Several forms of knowledge have been proposed to contribute to these classifications. Participants could either abstract the underlying rules [1], base their judgments on whole-item similarity to training exemplars [2], or base them on the relative familiarity of the fragments of which they are composed [3]. Rule-based classifications imply the formation of abstract mental representations which go beyond perceptual features. The exemplar account on the other hand assumes retrieval of specific training exemplars and judgments based on the global similarity between the test string and all the training strings. The fragment account is a perceptual-based theory of artificial grammar learning and postulates that participants learn about the frequency of occurrence of fragments of letters (i.e. two- or three-letter bigrams) and classify test strings as grammatical to the extent that they contain letter fragments that were present in the training strings. The presence of such fragments is assumed to cause perceptual priming, which in turn makes the string feel fluent.

There is continuing debate on what forms of knowledge are used to classify items in artificial grammar learning paradigms and under what conditions the different forms of knowledge make a contribution (see [4] for a review). The present study represents an attempt to contribute to this debate in a novel way via the collection of neuroimaging data. We test a simple prediction of the fragment-familiarity account: neural deactivation, a common correlate of repetition priming [5], should be observed for grammatical compared to ungrammatical stimuli.

Johnstone and Shanks devised a grammar, employed here, which allowed them to evaluate behaviourally the contributions of rule knowledge, exemplar similiarity, and fragment familiarity under more tightly-controlled conditions than is possible with traditional grammars [4] (see also $[6,7])$. Strikingly, Johnstone and Shanks found no evidence for a contribution of rule or exemplar knowledge under implicit learning conditions, but rather that participants classified the letter strings on the basis of fragment familiarity. In effect, the evidence suggested that participants 
become familiarized to string fragments (e.g. bigrams) in the study phase and then categorize test strings as grammatical to the extent that they are composed of familiar fragments. In other words, these behavioural results support a perceptually based account of categorization in artificial grammar learning.

Some neuroimaging studies have investigated neuronal correlates of category learning and found activity reductions in posterior occipital areas for categorical compared to noncategorical dot patterns [8-10]. These decreases were thought to reflect a perceptual priming effect in which processing of categorical items that share similarity with previously studied items is facilitated. The present study was performed to investigate whether a similar neuronal correlate was found for fragment-driven artificial grammar learning. In using the paradigm of Johnstone and Shanks [4] we expected to find differential activations to test items in occipital cortices as a function of the relationship between test items and study items. More specifically, given previous neuroimaging evidence [8], we expected to find occipital areas that would show reduced activity for test strings of high fragment familiarity.

\section{MATERIALS AND METHODS}

Subjects: Sixteen right-handed participants (nine male, seven female; age range 22-51 years, mean 30 years) with no history of medical or psychiatric disease gave informed consent to participate in the study. Exclusion criteria were $>20 \%$ missed responses during scanning (i.e. when no key press occurred until presentation of the next stimulus) or technical failure. This left data for 12 participants.

Stimuli: Stimuli were strings of eight pseudofont characters built from a vocabulary of six characters taken from [11]. Study stimuli were constructed according to a grammar which allowed only 18 of the possible 36 bigrams that can be built from the vocabulary (Fig. 1). Test stimuli comprised five classes each of 18 strings which systematically varied in terms of global similarity (i.e. exemplar familiarity) or fragment familiarity. Global exemplar similarity describes the similarity of the whole character string with a training string. Test items with high exemplar familiarity are strings which overlap in seven of the eight characters with training items whereas test items with low exemplar familiarity differ in $\geq 3$ letters from training items. Fragment familiarity relates to the number of two-character bigrams which a test string shares with a training string. Test items with high fragment familiarity were built of the 18 two-character bigrams which were used for construction of training items and thus had a high overlap in two- and three-character pseudofont fragments with training stimuli. Test items with low fragment familiarity on the other hand were built of the other 18 possible bigrams and thus had very low overlap in pseudofont character fragments with training stimuli (they also had low global similarity to the study strings).

Five different types of test stimuli were used: (1) old strings (OLD) were identical to study stimuli; (2) high exemplar/high fragment familiarity strings (HEHF) each overlapped globally with six study stimuli and also shared a high number of pseudofont character fragments; (3) low exemplar/high fragment familiarity strings (LEHF) differed
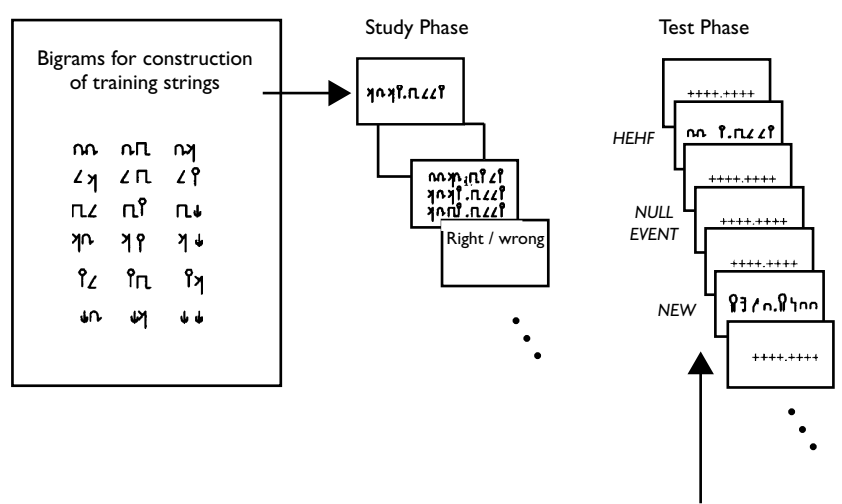

\begin{tabular}{|c|c|c|}
\hline \multicolumn{3}{|c|}{ Test strings } \\
\hline OLD & YnYi. $R \angle \angle 9$ & Repetition of study item \\
\hline HEHF & 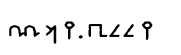 & $\begin{array}{l}\text { Similar as whole to } 6 \text { study items and composed } \\
\text { of familiar fragments }\end{array}$ \\
\hline LEHF & $9 \angle \angle \pi .97 n$ & $\begin{array}{l}\text { Not similar to any study items and but composed of familiar } \\
\text { fragments }\end{array}$ \\
\hline LELF & $n \angle \eta \pi . n \psi \pi \Pi$ & $\begin{array}{l}\text { Not similar to any study items and not composed of } \\
\text { familiar fragments }\end{array}$ \\
\hline NEW & 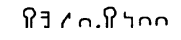 & LELF strings constructed from new symbols \\
\hline
\end{tabular}

Fig. I. Illustration of experimental paradigm, training and test stimuli. During a pre-scanning study phase participants performed a short term memory task on a series of pseudofont character strings. These training strings were of length eight and constructed from a vocabulary of six characters. All strings were constructed of 18 (see box in lower left of the figure) of the possible 36 bigrams that can be build from the vocabulary, thus familiarizing participants to string fragments. Test strings were also built of eight pseudofont characters and varied systematically in their similarity to training strings (OLD, HEHF, LEHF, LELF, NEW). An example of a test string of each stimulus type is given (see box in lower right of the figure). Null events (i.e. imaginary events which are not different from baseline) were randomly intermixed with training strings. Stimuli were randomly displayed every $4 \mathrm{~s}$ for $2 \mathrm{~s}$ against a baseline of fixation crosses and participants had to decide with a keypress whether the string conformed to the grammar.

from all study stimuli in at least three pseudofont characters and therefore had low global similarity but comprised a large number of familiar pseudofont character fragments; (4) low exemplar/low fragment strings (LELF) had low global similarity and had a minimal overlap in pseudofont character fragments; (5) new strings (NEW) were built from a different vocabulary of pseudofont characters. For this purpose characters in the LELF strings were replaced with new pseudofont characters. Note that HELF items cannot be constructed because it is not possible for strings to be similar to studied exemplars yet not be composed of familiar fragments. Participants also performed a parallel version of the task using strings built of letters rather than pseudofont characters. These data are not presented since no posterior brain regions showed stimulus effects in the F-test (further details available on request). If grammaticality decisions are driven by fragment familiarity then we expect to see strings composed of familiar fragments (OLD, HEHF, LEHF) endorsed as grammatical more than ones composed of unfamiliar fragments (LELF, NEW). If global similarity is 
the main determinant of decisions [2] then we expect to see strings with high global similarity (OLD, HEHF) endorsed as grammatical more than ones with low global similarity (LEHF, LELF, NEW).

Experimental design (see also [4], experiment 5): In a study phase, conducted outside the scanner, subjects were presented visually with training strings (Fig. 1). On each of 108 trials a string was displayed for 7s on a Macintosh computer and participants were asked to mentally rehearse it. Then the screen went blank for $2 \mathrm{~s}$ following which a list of three strings (numbered 1-3) was displayed and the participant was asked to type the number of the string that matched the one they were rehearsing (using the keys for numbers $1-3$ on the computer keyboard). The two foils were illegal versions of the correct string. The order of training strings was randomised across participants.

Scanning took place during a subsequent test phase. Participants were taken directly from the training room to the scanner room. The approximate delay between the end of the test phase and the start of scanning was $20 \mathrm{~min}$. Inside the scanner participants were presented with the five different types of test strings described above for $2 \mathrm{~s}$ every $4 \mathrm{~s}$ on a Macintosh computer against a baseline of crosshairs, projected onto a screen $\sim 300 \mathrm{~mm}$ above the participant in the MRI scanner. The stimulus presentation included null events, where a baseline picture is presented instead of a stimulus (see also Fig. 1 for illustration). These null events were randomly intermixed with the test stimuli and made up one-third of events (i.e. 45 null events for 135 stimuli). Note that such null events are completely undetectable by subjects. The inclusion of null events is used to achieve a random distribution of SOAs and is equivalent to the use of a variable interstimulus baseline. Null events are included in event-related fMRI experiments to increase baseline periods and thus the sensitivity for the main effect (i.e. all stimulus types vs baseline) even with short SOAs [12]. Volunteers were informed that the strings they had been asked to memorize previously were generated from a complex set of rules (the rule being that the strings were composed of a subset of only 18 of the 36 possible bigrams) and were asked to classify the strings encountered during scanning as grammatical or ungrammatical depending on whether they appeared to conform to the rules or not by pressing one of two response keys. They were told not to worry if they did not notice any rules and to make an instinctive judgment.

Data acquisition: A Vision MRI system (Siemens, Erlangen, Germany) operating at $2 \mathrm{~T}$ was used to obtain $\mathrm{T} 2$ *weighted echoplanar $(E P I)$ images (in plane matrix $64 \times 64$ ) with blood oxygenation level dependent (BOLD) contrast. In each session 225 volumes of $302 \mathrm{~mm}$ axial slices were acquired sequentially every $3.5 \mathrm{~mm}$ (voxel size $3 \times 3 \times 3.5 \mathrm{~mm}$ pixels, repetition time $2.5 \mathrm{~s}$, echo time $40 \mathrm{~ms})$. The first five volumes were discarded to allow for $\mathrm{T} 1$ equilibration effects. Images were spatially realigned to the first volume to correct for interscan movement, synchronized to the middle slice to correct for differences in slice acquisition time, and normalized to a standard EPI template volume (resampled to $3 \times 3 \times 3 \mathrm{~mm}^{3}$ voxels). The data were then smoothed with a Gaussian kernel of $8 \mathrm{~mm}$ full-width at half maximum to accommodate intersubject anatomical variability.

Statistical analyses of imaging data: Data were analysed with Statistical Parametric Mapping software (SPM99, Wellcome Department of Cognitive Neurology, London) [13] employing a random effects model. At the first level six event types were defined. These consisted of five effects of interest (OLD, HEHF, HILA, LELF, NEW) and one effect of no interest (missed responses). The event types were modelled by a canonical synthetic haemodynamic response function (HRF) and its first-order temporal derivative. The six head movement parameters were included as confounds. Data were globally scaled to 100 across scans and highpass-filtered at $1 / 60 \mathrm{~Hz}$. The parameter estimates for the canonical HRF and linear contrasts of these estimates comprised the data for the second-stage of analyses. At the second stage, a statistical parametric map (thresholded at $p<0.001$ ) of voxels showing significant responses to stimulus presentation versus baseline (i.e. main effect of task: $\quad[(\mathrm{OLD}+\mathrm{HEHF}+\mathrm{HILA}+\mathrm{LELF}+\mathrm{NEW})>$ baseline $])$ was created. Note that the baseline included the null events. This mask, which identified regions responsive to presentation of pseudofont character strings, was then used to identify brain regions differentially responsive to stimulus similarity according to two planned comparisons. First, an omnibus F-test, comprising the five effects of interest, identified regions differentially active as a function of stimulus type. Activations are reported at a level of significance $p<0.001$, uncorrected. The effect maximum was plotted and analysed with four paired $t$-tests (OLD vs HEHF, HEHF vs LEHF, LEHF vs LELF and LELF vs NEW). $p<0.05$ was regarded as significant (after Bonferroni correction for four pairwise comparisons). Second, a $t$-test, identifying regions which show reduced activation as a function of fragment familiarity [LELF-(HEHF + LEHF)], based on the predicted pattern of behavioural data [4]. Activations are reported at a level of significance of $p<0.001$, uncorrected. Even though only three stimulus types went into this SPM analysis, effect maxima were plotted for all stimulus types for reasons of illustration. The fragment familiarity effect was analysed with one paired $t$-test (LEHF vs LELF), based on behavioural data. $p \leq 0.05$ was regarded as significant.

Statistical analyses of behavioural data: Differences in grammaticality judgments and reaction times between all five test items were analysed with ANOVA for repeated measures (with Greenhouse-Geisser correction). Significant effects were followed by two planned $t$-tests (with Bonferroni correction), one for fragment familiarity-based judgments (LEHF vs LELF) and one for global similarity-based judgments (HEHF vs LEHF).

\section{RESULTS}

Behavioural data: The mean percentage of training trials on which participants made correct responses during the study phase was $98 \%$. Table 1 shows the percentage of test strings classified as grammatical and the associated mean reaction times. We found a significant main effect of 
stimulus type $(F(1.9,20.6)=9.40, p M<0.005)$ with higher classifications of LEHF strings as grammatical than LELF strings $(t(11)=3.2, p<0.05)$. There was no difference between HEHF strings and LEHF strings $(\mathrm{t}(11)=-0.160$, $p=0.876$. The data thus confirm that participants made grammaticality judgments on the basis of fragment familiarity (LEHF vs LELF) and not on the basis of whole-item similarity (HEHF vs LEHF). Reaction time data did not show an effect of stimulus type $(\mathrm{F}(1.3,15.0)=1.19, p=0.292)$. The imaging data thus allow us to ask whether the predicted pattern of neural deactivation typical of perceptual priming underlies this fragment familiarity effect.

Imaging data: A network of regions including left and right extrastriate areas, bilateral inferior frontal gyri, bilateral inferior parietal lobules, left motor cortex and anterior cingulate were activated (at $p<0.001$, uncorrected) when event-related responses to all string-types were compared to the interstimulus baseline. Within this network, responsive to presentation of pseudofont stimuli, we first identified, using an F-test, regions differentially active as a function of stimulus type (Fig. 2). A differentially responsive extrastriate brain region was found in left fusiform gyrus $(x=-45, y=-60, z=-27,21$ voxels, $\mathrm{Z}=4.04, \quad p<0.001$ uncorrected). The right hand graph shows percentage signal change for the five different stimuli in this region. Fusiform responses were higher for NEW strings than the other types of strings (there was also a trend for lower responses to OLD strings than HEHF, LEHF and LELF items, though this did not reach significance). Two smaller voxel clusters showing differential activation to stimulus type were found in left lateral occipital complex $(\mathrm{x}=-39, \mathrm{y}=-90, \mathrm{z}=-9,5$ voxels, $\mathrm{Z}=3.55, p<0.001$ uncorrected) and right occipital medial gyrus $(x=33$, $\mathrm{y}=-84, \mathrm{z}=21,3$ voxels, $\mathrm{Z}=3.38, p<0.001$ uncorrected). Note that differential activity was also evident in left inferior parietal cortex (BA40, $\mathrm{x}=-42, \mathrm{y}=-42, \mathrm{z}=54,25$ voxels, $\mathrm{Z}=4.58, p<0.001$ uncorrected). Responses in this region also showed the numerically highest responses to NEW strings and the lowest responses to OLD strings. In addition, greater responses to HEHF than to OLD and LEHF strings were observed (data not shown).

Brain areas showing a sensitivity to our planned comparison of fragment familiarity are shown in Fig. 3. Regions showing reduced activations to strings with high fragment familiarity as compared to strings with low fragment familiarity [LELF-(HEHF + LEHF)] included the left lateral occipital complex (BA19, $x=-39, y=-87$, $\mathrm{Z}=-9,10$ voxels, $\mathrm{Z}=4.35, p<0.001$ uncorrected) and a bilateral posterior occipital region comprising the lingual gyri (BA 18, $x=-12, y=-87, \quad z=-15$, eight voxels, $\mathrm{Z}=3.58, \quad p \leq 0.001$ uncorrected and $\mathrm{x}=15, \quad \mathrm{y}=-81$ $\mathrm{Z}=-[1] 2,4$ voxels, $\mathrm{Z}=3.41, p<0.001$ uncorrected). Note that the cluster of voxels in left lateral occipital complex was the same as the cluster of voxels in left lateral occipital cortex identified by the F-test. Percentage signal change

Table I. Items identified as grammatical.

\begin{tabular}{lcccc}
\hline & OLD & HEHF & LEHF & LELF \\
\hline Judgement & $74 \pm<4 \%$ & $74 \pm<4 \%$ & $74 \pm<5 \%$ & $57 \pm<6 \%$ \\
Reaction times & $1803 \pm<134 \mathrm{~ms}$ & $1874 \pm<101 \mathrm{~ms}$ & $1978 \pm<164 \mathrm{~ms}$ & $1896 \pm<141 \mathrm{~ms}$ \\
\hline
\end{tabular}

Percentage items identified as grammatical judgement and reaction times shown as mean \pm s.e.m.
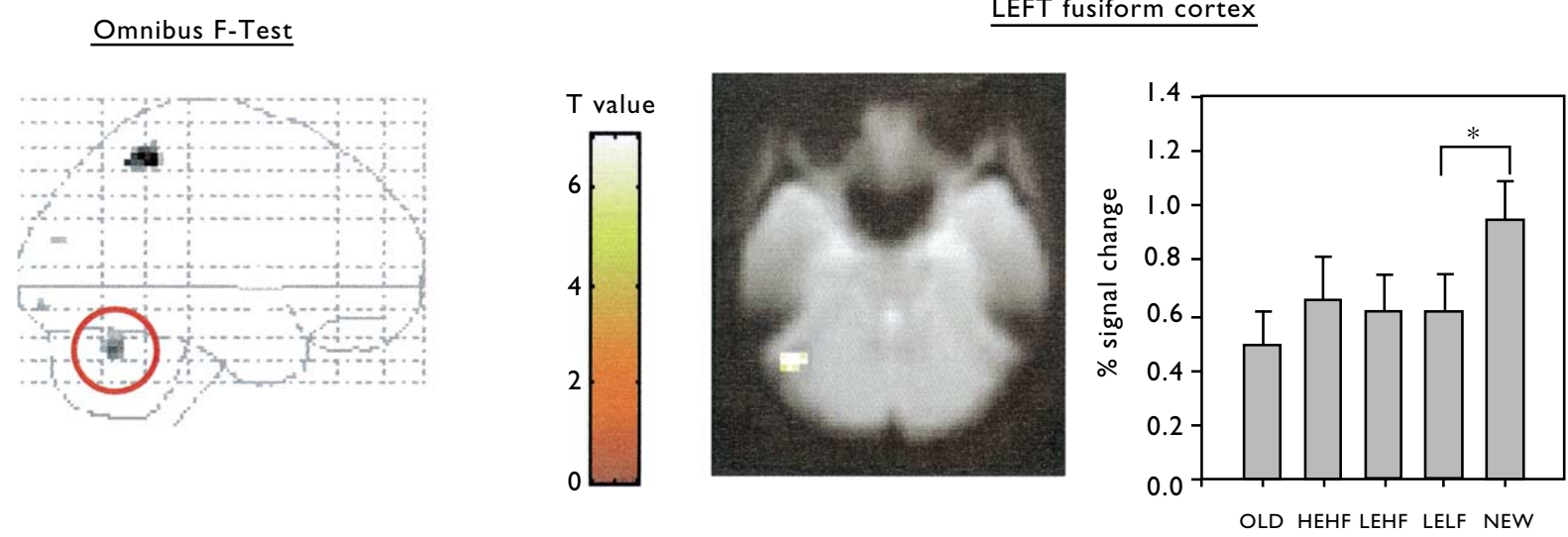

Fig. 2. Regions differentially active as a function of stimulus type (F-test). Activations are found in left fusiform cortex (rendered on transverse mean normalized EPI images). EPI images are used for anatomical descriptions, since they show the same distortions as activation data. EPI images were averaged over several volunteers and activations thresholded at $p<0.001$, uncorrected. Plots of percentage signal change are shown for the maximally activated voxel $(x=-45, y=-60, z=-27)$. The plot illustrates the activations for the different stimulus types. Data were analysed with ANOVAs for repeated measures followed by four paired $t$-tests (Bonferroni corrected; ** $p<0.01$, * $p<0.05$ ). 
LELF-(HEHF+LEHF)

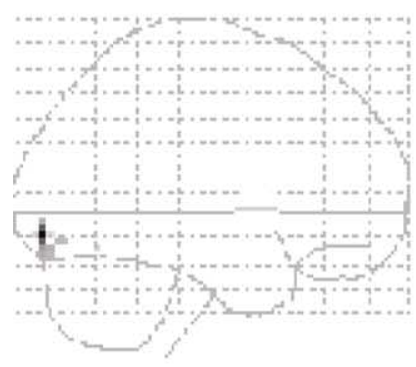

$z=-15 \mathrm{~mm}$

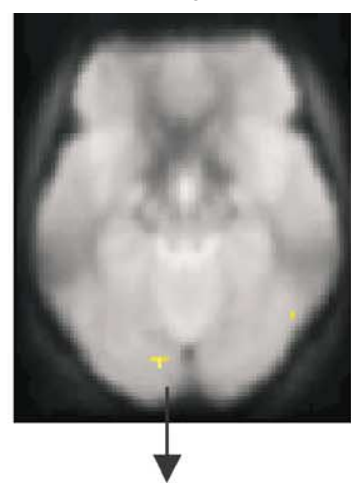

left lingual gyrus $(-12-87-15)$
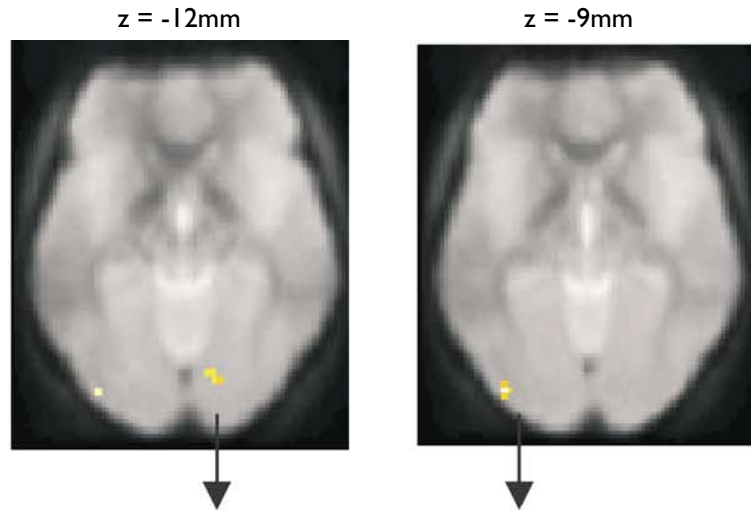

right lingual gyrus $(-15-81-12)$

left lateral occipital complex (-39-87 -9)
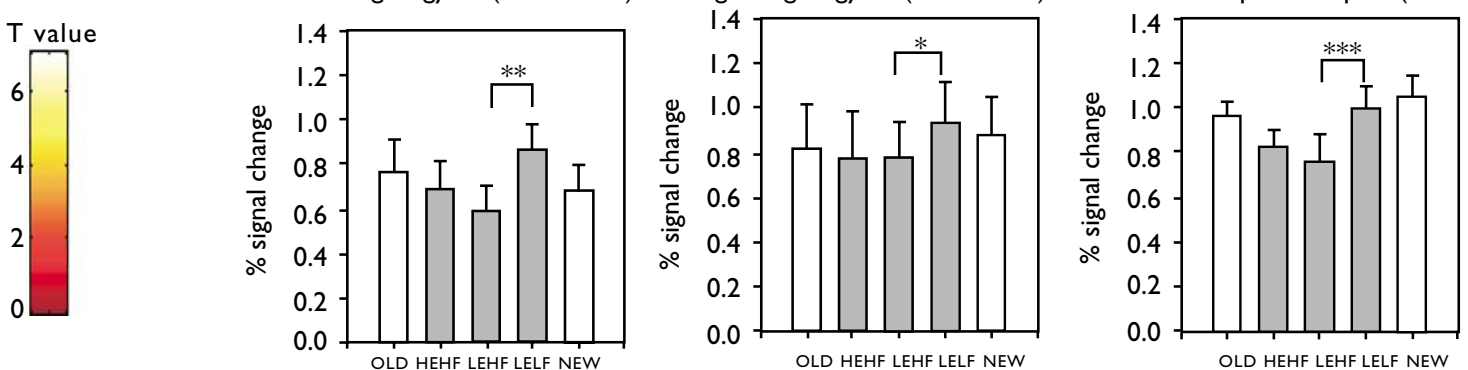

Fig. 3. Fragment familiarity effects [LELF-(HEHF + LEHF)]. Lower activations to strings with high (HEHF and LEHF) as compared to ones with low fragment familiarity (LELF) were evident in bilateral lingual gyri and left lateral occipital complex. Activations $(p<0.001$, uncorrected) are shown on a glass brain and mean normalized EPI slices. Plots of percentage signal change are shown for the maxima of these brain regions and illustrate the neuronal facilitation depending on fragment familiarity (grey bars). For purposes of illustration percentage signal change in these regions was also plotted for NEW and OLD strings (white bars). Data were analysed with ANOVAs for repeated measures followed by one paired $t$-test for each region. $* * * p<0.00 I$, $* * p<0.01$, $* p<0.05$.

plotted for all stimuli in these brain regions demonstrated the differential sensitivity to fragment familiarity with higher responses to strings with low (LELF) than to high fragment familiarity (LEHF and HEHF). HEHF, LEHF and LELF items which constitute the SPM analysis are highlighted in grey; OLD and NEW items are plotted for illustration.

\section{DISCUSSION}

We investigated fMRI correlates of fragment-based knowledge in an artificial grammar learning paradigm using strings of pseudofont characters. Fragment familiarity effects, which we interpret in terms of priming, were evident in behavioural and imaging data. In particular, activity reductions occurred in occipital brain regions to items with high fragment familiarity. These findings are consistent with the idea that one correlate of categorization in grammar learning paradigms involves a perceptual process which is evident as neuronal facilitation based in extrastriate visual regions.

Our behavioural data replicate experimental findings by Johnstone and Shanks [4] and show that participants classify strings on the basis of fragment familiarity rather than global exemplar similarity. The behavioural results demonstrate that such fragment familiarity-driven classifications hold not only for strings built out of letter characters (as shown in previous studies) but also for strings constructed from pseudofont characters. This is consistent with the conjecture that participants become familiarized to the fragments (e.g. bigrams) of which the training strings are composed and then classify test items according to the familiarity of their component parts.

The left fusiform region showed lower responses to pseudofont character strings that were similar or identical to training items (OLD, HEHF, LEHF and LELF) than strings consisting of new pseudofont characters (NEW). No significant difference was found between strings that showed subtle variations in familiarity in terms of fragment or exemplar similarity. These data suggest that the left fusiform can exhibit some form of generalisation over changes in stimulus form such that greater relative responses are only seen to novel items (or strings comprising novel combinations of familiar components). A certain kind of generalisation over stimulus form has been shown in fusiform cortex in other studies. Roission and colleagues presented volunteers with faces that had been morphed along a continuum from pre-exposed to novel and showed categorical activation patterns in right occipito-temporal regions, including the right fusiform gyrus and middle occipital gyrus [14]. These were evident as a reduced response for faces perceived as familiar (60-100\% morphs of pre-exposed faces), relative to faces perceived as unfamiliar (0-40\% morphs), even though there were subtle variations in familiarity within the familiar and unfamiliar faces. It also resembles findings that activation decrease in the left fusiform area to pre-exposed objects is invariant to changes in the size or view of those objects [15-17]. 
Several previous studies identified neural correlates of acquisition of a finite state grammar [18]. The only study that investigated grammatical classifications after the grammar had been learned was performed by Seger et al. [8]. They compared grammaticality judgments in a block of grammatical letter strings with bigram detection in another block of letter strings, and found activations in bilateral occipitoparietal cortices and left dorsolateral prefrontal cortex as well as deactivation in precuneus [19]. Such a comparison does not determine, however, what form of knowledge (abstract rules, exemplar information, or fragment familiarity) is involved in classification performance. Here we present evidence of differential activity to strings perceived as grammatical (based on fragment familiarity) and those perceived as ungrammatical in conditions where it is known that fragment familiarity is the key source driving classification decisions.

Areas sensitive to fragment familiarity were found in posterior occipital regions including the left lateral occipital complex and bilateral lingual gyri. In these regions, lower responses were observed to test strings that shared a high number of fragments (HEHF, LEHF) with training strings as compared to those that shared a low number of fragments (LELF). The lateral occipital complex is a region critical for object recognition, dependent on higher level shape information [16,20-22]. The differential activations reported here fall within the posterior lateral occipital complex, which has been found to show repetition-related response decreases to objects with some sensitivity to changes in position and size [17]. Response reductions to strings with high fragment similarity were also evident in bilateral lingual gyri close to the areas in posterior occipital cortices previously reported during classification of categorical as opposed to non-categorical dot patterns [8,9]. Thus, while fusiform regions appeared sensitive to completely new strings, yet not significantly modulated by the similarity to pre-exposed strings, the occipital regions were modulated by fragment familiarity of pre-exposed strings.

Our results extend the findings by Reber and colleagues, which showed activity reductions in posterior occipital areas during processing of dot patterns that shared overall similarity with previously studied dot patterns. Our data, in which exemplar and fragment similarity can be dissociated, indicate that facilitation of neuronal processing in these posterior occipital regions reflects fragment familiarity of items. From a behavioural perspective the data suggest that neuronal facilitation of items with high fragment similarity, resulting in perceptual priming, may provide one neuronal substrate for grammaticality classifications in artificial grammar learning paradigms. Future work might usefully study conditions in which forms of knowledge other than fragment familiarity may make more of a contribution to classification performance and may identify, for instance, brain regions involved when abstract rule knowledge is deployed in making classification decisions [23].

\section{CONCLUSIONS}

We demonstrated that extrastriate occipital brain areas show decreased responses to pseudofont character strings that share a high number of fragments with previously presented strings. The observed response decreases might therefore constitute one possible neuronal mechanism for category learning of unfamiliar letter-like stimuli. The observation of neural reductions associated with grammaticality judgments adds confirmatory weight to the claim that such judgments are based on the experienced fragment familiarity of the test items via a process of perceptual priming. Our results represent a compelling example of neuroimaging data providing converging and complementary evidence for a theory otherwise only supported behaviorally. Further studies are needed to investigate whether extrastriate response decreases are a general signature of category learning or specific to the stimulus material used.

\section{REFERENCES}

1. Reber AS. J Exp Psychol Gen 118, 219-235 (1989).

2. Vokey JR and Brooks LR. J Exp Psychol Learn Mem Cogn 18, 328-344 (1992).

3. Perruchet P and Pacteau C. J Exp Psychol Gen 119, 264-275 (1990).

4. Johnstone T and Shanks DR. Cogn Psychol 42, 61-112 (2001).

5. Schacter DL and Buckner RL. Neuron 20, 185-195 (1998).

6. Johnstone T and Shanks DR. J Exp Psychol Learn Mem Cogn 25, 524-531 (1999).

7. Kinder A and Assmann A. Mem Cogn 28, 1321-1332 (2000).

8. Reber PJ, Stark CE and Squire LR. Learn Mem 5, 420-428 (1998).

9. Reber PJ, Stark CE and Squire LR. Proc Natl Acad Sci USA 95, 747-750 (1998).

10. Aizenstein HJ, MacDonald AW, Stenger VA et al. J Cogn Neurosci 12, 977987 (2000).

11. Howard D, Patterson K, Wise R et al. Brain 115, 1769-1782 (1992).

12. Josephs O and Henson RN. Phil Trans R Soc Lond B Biol Sci 354, 1215-1228 (1999).

13. Friston KJ, Holmes AP, Worsley KJ et al. Hum. Brain Mapp 2, 189-210 (1995).

14. Rossion B, Schiltz C, Robaye L et al. J Cogn Neurosci 13, 1019-1034 (2001).

15. Vuilleumier P, Henson RN, Driver J et al. Nature Neurosci 5, 491-499 (2002).

16. Kourtzi Z and Kanwisher N. J Neurosci 20, 3310-3318 (2000).

17. Grill-Spector K, Kushnir T, Edelman S et al. Neuron 24, 187-203 (1999).

18. Fletcher P, Buchel C, Josephs O et al. Cerebr Cortex 9, 168-178 (1999).

19. Seger CA, Prabhakaran V, Poldrack RA et al. Psychobiology 28, 283-292 (2001).

20. Grill-Spector K, Kourtzi Z and Kanwisher N. Vision Res 41, 1409-1422 (2001).

21. Lerner Y, Hendler T, Ben Bashat D et al. Cerebr Cortex 11, 287-297 (2001).

22. Kourtzi Z and Kanwisher N. Science 293, 1506-1509 (2001).

23. Smith EE, Patalano AL and Jonides J. Cognition 65, 167-1961 (1998).

\footnotetext{
Acknowledgements: We thank radiographers for help with fMRI scanning. This work was supported by a Wellcome Trust programme grant to R.J.D., a WellcomeTrust Fellowship to RN.A.H., a research grant from the Economic and Social Research Council to D.R.S. and a research grant from the German Research Foundation (DFG) to C.M.T.
} 